\title{
Uptake of tenofovir and emtricitabine into non-monocytic female genital tract cells with and without hormonal contraceptives
}

This article was published in the following Dove Press journal:

Journal of Experimental Pharmacology

I August 2013

Number of times this article has been viewed

\author{
Amanda Marie James' \\ Jennifer R King' \\ Ighovwerha Ofotokun ${ }^{2}$ \\ Anandi N Sheth ${ }^{2}$ \\ Edward P Acosta \\ 'Division of Clinical Pharmacology, \\ 2Emory University School of \\ Medicine, Department of Medicine, \\ Division of Infectious Diseases, The \\ University of Alabama at Birmingham, \\ Birmingham, AL, USA
}

Background: Pre-exposure prophylaxis is becoming a strategic component used to control the human immunodeficiency virus (HIV-1) epidemic. The goal of this study was to characterize intracellular uptake of tenofovir and emtricitabine using five surrogate cell lines of the female genital tract and determine whether exogenous hormones influence their uptake.

Methods: Surrogate cell lines, ie, THP-1 (representing macrophages), BC-3 (CD8+), Ect1/ E6E7 (squamous epithelial), HeLa (CD4+), and TF-1 (dendritic), were incubated for one hour with tenofovir and emtricitabine to assess uptake. In separate experiments, ethinyl estradiol (EE) and etonogestrel (ET) individually and together (EE/ET) were added prior to, simultaneously, and after incubation. Intracellular phosphorylated tenofovir and emtricitabine were quantified using validated tandem mass spectrometry methods.

Results: HeLa and Ect1/E6E7 cells showed significantly increased uptake relative to THP-1 controls for both antiretrovirals. Individually, ethinyl estradiol and etonogestrel significantly altered antiretroviral uptake across all cell lines, except Ect1/E6E7 for tenofovir and HeLa for emtricitabine. Cellular uptake of tenofovir and emtricitabine in BC-3 and TF-1 cells were significantly lower when dosed one hour prior to EE/ET administration compared with each antiretroviral administered in the absence of EE/ET (tenofovir, 80 versus $470 \mathrm{fmol} / 10^{6}$ for BC-3 and 77 versus 506 fmol/ $10^{6}$ cells for TF-1; emtricitabine, 36 versus 12 fmol/10 for BC-3 and 75 versus $5 \mathrm{fmol} / 10^{6}$ cells for TF-1; $P<0.01$ for each).

Conclusion: These data suggest that intracellular uptake of tenofovir and emtricitabine within the female genital tract varies by cell type and in the presence of hormonal contraceptives. The potential clinical implications of these findings should be further evaluated in vivo.

Keywords: pre-exposure prophylaxis, tenofovir, emtricitabine, in vitro, hormones

\section{Introduction}

The use of antiretrovirals by individuals negative for human immunodeficiency virus (HIV) to prevent HIV-1 infection, also known as pre-exposure prophylaxis (PrEP), has shown variable results in reducing the rate of HIV-1 transmission. ${ }^{1,2}$ The Center for the AIDS Program of Research in South Africa 004 trial, which examined topical $1 \%$ tenofovir gel in women, showed a $39 \%$ overall reduction in HIV-1 transmission. ${ }^{3}$ The PrEP Initiative study demonstrated a 42\% decrease in HIV-1 transmission when daily oral tenofovir disoproxil fumarate (TDF) and emtricitabine were administered to men who have sex with men, ${ }^{4}$ while the TDF2 and Partners PrEP studies demonstrated over $60 \%$ efficacy against acquisition of HIV-1 in discordant heterosexual men and women when daily TDF was used with or without emtricitabine. ${ }^{5,6}$ In contrast, no significant difference was demonstrated between TDF and placebo
Division of Clinical Pharmacology, University of Alabama at Birmingham, School of Medicine, 1670 University Blvd, Volker Hall, Room 258, Birmingham, AL 35294-0019, USA

Tel +I 2059342655

Fax + I 205934620 I

Email eacosta@uab.edu which permits unrestricted noncommercial use, provided the original work is properly cited. 
in their ability to prevent HIV-1 infection in participants enrolled in the Vaginal and Oral Interventions to Control the Epidemic trial. ${ }^{7}$ Similarly, an oral combination of TDF and emtricitabine was not able to demonstrate effectiveness in preventing HIV-1 infection in HIV-negative women enrolled into the FEM-PrEP study who were at high risk of being exposed to $\mathrm{HIV}-1 .{ }^{8}$ As a result, these two PrEP trials were prematurely terminated.

Differences in outcomes between prevention trials could be due to several factors. Each study evaluated once-daily administration of TDF with or without emtricitabine, but the routes of administration differed. The data suggest that vaginal administration produces 100 times greater tenofovir concentrations in endocervical cells than oral dosing, thus possibly providing better protection against transmission of HIV-1. ${ }^{9}$ Adherence to therapy likely also plays an important role, because studies have reported $>90 \%$ adherence by participants, whereas antiretroviral plasma concentrations suggest only $50 \%$ adherence. ${ }^{10}$ Finally, concomitant use of hormonal contraceptives was not an exclusion criterion. Although oral hormonal contraceptives do not alter the plasma pharmacokinetics of tenofovir or emtricitabine, ${ }^{11}$ tenofovir and emtricitabine require intracellular phosphorylation to the active diphosphate or triphosphate moieties, respectively. ${ }^{12,13}$ The possibility that hormones influence cellular uptake of nucleoside reverse transcriptase inhibitors has not been extensively evaluated. ${ }^{14}$

These factors support integrating intracellular pharmacology into clinical evaluations of PrEP studies. Several studies to date have quantified intracellular concentrations of tenofovir and emtricitabine in the female genital tract to determine the concentrations required to prevent HIV-1 transmission. Analytical methods to quantify intracellular concentrations in this compartment may differ, potentially leading to inconsistent results across studies. For example, most methods use macrophage-like peripheral blood mononuclear cells as controls and standards. However, cells of the female genital tract also include squamous epithelial, dendritic, CD4+, and CD8+ cells. ${ }^{15-17}$ The female genital tract can be divided into five major sections, ie, the vagina, cervix, uterus, fallopian tubes, and ovaries. The cervix and vagina are commonly sampled to quantify antiretrovirals because these sections are highly vulnerable to HIV-1 transmission and collection techniques, such as cytobrushing, direct aspirate, and TearFlo strips that target cells in these areas. ${ }^{18}$ Therefore, antiretroviral uptake in cell lines that match cell types in the endocervix, ectocervix, and vagina should be evaluated. ${ }^{19-21}$
Our hypotheses were that uptake of tenofovir and emtricitabine into these surrogate cell lines would not differ, and that administration of ethinyl estradiol and/or etonogestrel would not alter intracellular anabolite concentrations. The objectives of this study were to assess intracellular concentrations of tenofovir and emtricitabine in THP-1 cells, ${ }^{22}$ which represent macrophages, and four surrogate cell lines of the female genital tract, ie, BC-3 (representing CD8+ cells), Ect1/E6E7 (squamous epithelial cells), HeLa (CD4+ cells), and TF-1 (dendritic cells), ${ }^{23-26}$ and to compare intracellular tenofovir and emtricitabine concentrations across these surrogate cell lines when dosed prior to, simultaneously, and after ethinyl estradiol and/or etonogestrel.

\section{Materials and methods Materials}

Tenofovir and emtricitabine were obtained for laboratory use only from the National Institutes of Health AIDS Research and Reagent Program. Ethinyl estradiol was purchased from Sigma-Aldrich (St Louis, MO, USA) and etonogestrel from Santa Cruz Biotechnology Inc (Santa Cruz, CA, USA). Isotopically-labeled tenofovir and emtricitabine were purchased from Moravek Inc (Brea, CA, USA) and used as the internal standard. All additional solvents were purchased from commercial suppliers and were of high performance liquid chromatography grade. The five different cell lines (THP-1, HeLa, BC-3, TF-1, and Ect1/E6E7) were obtained from the American Type Culture Collection (Rockville, MD, USA) and grown in medium prepared in accordance with American Type Culture Collection protocols in a freestanding Isotemp incubator (Fisher Scientific, Waltham, MA, USA) at $37^{\circ} \mathrm{C}$ with $5 \% \mathrm{CO}_{2}$. The cells were passaged between five and 12 times at $95 \%-100 \%$ confluence. Confluent monolayers of adherent cell lines were subcultured by treatment with $0.35 \%$ trypsin containing EDTA, while confluent monolayers of suspension cell lines were subcultured with a one to three splitting technique.

\section{Cell dosing studies}

\section{Uptake of tenofovir and emtricitabine \\ in the absence of hormones}

Each cell line was dosed (with six replicates) using either $0.3 \mu \mathrm{g} / \mathrm{mL}$ of tenofovir or $1.8 \mu \mathrm{g} / \mathrm{mL}$ of emtricitabine, representing the peak plasma concentration $\left(\mathrm{C}_{\max }\right)$ recorded in the literature for adults. ${ }^{27,28}$ After one hour, the cells were harvested by removing the medium and pelleted using centrifugation $\left(5000 \mathrm{rpm}\right.$ for 10 minutes at $\left.25^{\circ} \mathrm{C}\right)$. The pellet was resuspended in $70 \%$ methanol, vortexed, placed on ice for 
15 minutes, and centrifuged. The supernatant, representing the cellular matrix, was collected and stored at $-80^{\circ} \mathrm{C}$ until analysis. Cell viability was checked prior to and after dosing by cell count, visual inspection, and the Trypan Blue Cell Viability test. Interday variability was also assessed.

\section{Uptake of tenofovir and emtricitabine in the presence of hormones}

Each cell line (5-6 replicates) was dosed with $0.3 \mu \mathrm{g} / \mathrm{mL}$ of tenofovir or $1.8 \mu \mathrm{g} / \mathrm{mL}$ of emtricitabine. However, in this set of experiments, the cells were also dosed with $34.7 \mathrm{pg} / \mathrm{mL}$ of ethinyl estradiol and/or $1716 \mathrm{pg} / \mathrm{mL}$ of etonogestrel, representing the plasma $\mathrm{C}_{\max }$ recorded in the literature for the NuvaRing ${ }^{\circledR}$ contraceptive (Merck, Whitehouse Station, NJ, USA). ${ }^{29}$ Dosing experiments with ethinyl estradiol and/or etonogestrel were conducted relative to the time of tenofovir and emtricitabine dosing. All cell lines were dosed with either tenofovir or emtricitabine one hour prior to, simultaneously, or one hour after dosing with the hormonal contraceptives. In each case, the hormones were allowed to incubate with the drug for one hour. The effect of EE/ET on antiretroviral uptake across a 25-fold range of tenofovir and emtricitabine doses was also examined. In addition to the initial doses of $0.3 \mu \mathrm{g} / \mathrm{mL}$ of tenofovir and $1.8 \mu \mathrm{g} / \mathrm{mL}$ of emtricitabine, the cells were also dosed with a concentration representing a minimum plasma concentration $\left(\mathrm{C}_{\text {min }}\right)$ of $0.06 \mu \mathrm{g} / \mathrm{mL}$ for tenofovir and $0.09 \mu \mathrm{g} / \mathrm{mL}$ for emtricitabine and a 25 -fold increase from the $\mathrm{C}_{\text {min }}$ of $1.5 \mu \mathrm{g} / \mathrm{mL}$ for tenofovir and $2.25 \mu \mathrm{g} / \mathrm{mL}$ for emtricitabine. All samples were processed as described in the previous paragraph.

\section{Sample preparation and mass spectrometry}

Cellular lysate samples were subjected to anion exchange solid phase extraction with a QMA cartridge (Accell Plus QMA, 3 mL, Waters Corporation, Milford, MA, USA) using a potassium chloride salt gradient of 5-100 $\mathrm{mM}$ to isolate the phosphorylated forms of the drugs. The samples were then dephosphorylated from the active tenofovir diphosphate and emtricitabine triphosphate to measurable tenofovir and emtricitabine using sweet potato acid phosphatase XA (Sigma-Aldrich)/acetate buffer at $37^{\circ} \mathrm{C}$ for one hour. Cellular lysate containing only tenofovir and emtricitabine was then desalted by a solid phase extraction with a reverse phase Oasis HLB cartridge (3 $\mathrm{mL}$, Waters Corporation). Desalted cellular lysate samples were then evaporated under nitrogen at $37^{\circ} \mathrm{C}$ and reconstituted in double-distilled $\mathrm{H}_{2} \mathrm{O}$ to concentrate the samples. Quantification of tenofovir and emtricitabine was performed using a method developed on a Prominence Ultra-Fast liquid chromatography system (UFLC $_{\mathrm{XR}}$, Shimadzu, Tokyo, Japan) coupled to an MDS Sciex API 5500 triple quadrupole mass spectrometer. Chromatographic separation was performed on an Atlantis $\mathrm{dC} 18,2.1 \times 100 \mathrm{~mm}, 3 \mu \mathrm{m}$ particle size, analytical column (Waters Corporation). The mobile phase consisted of $0.01 \%$ trifluoroacetic acid buffer and acetonitrile run isocratically at a ratio of 90:10 (by volume). Detection and quantification were achieved by multiple reaction monitoring. Tenofovir, tenofovir-internal standard, emtricitabine, and emtricitabineinternal standard were detected using the following transitions for protonated daughters $[\mathrm{M}+\mathrm{H}]^{+}: \mathrm{m} / \mathrm{z}$ tenofovir $288 \rightarrow 176.1$ and $288 \rightarrow 159, \mathrm{~m} / \mathrm{z}$ tenofovir-internal standard $293 \rightarrow 181, \mathrm{~m} / \mathrm{z}$ emtricitabine $248.1 \rightarrow 130$ and $248.1 \rightarrow 113, \mathrm{~m} / \mathrm{z}$ emtricitabineinternal standard $251 \rightarrow 133$. The analytical concentration range for intracellular tenofovir and emtricitabine was 5-2500 fmol $/ 10^{6}$ cells. Standard curves, quality controls, and blanks for each cell line were prepared to ensure accurate quantitation throughout the analysis. Acceptance criteria for the assay were based on Good Clinical Laboratory Procedures standards, which consisted of standard curves and quality controls within $\pm 15 \%$ of their nominal value along with the blank of each cell line demonstrating no measurable contamination response from preparation of samples.

\section{Data analysis}

THP-1 cells were used as the initial control cell line for statistical comparisons with the other cell lines using the unpaired nonparametric $t$-test (Figure 1). Uptake of tenofovir or emtricitabine from each cell line dosed with ethinyl estradiol and/or etonogestrel prior to, simultaneously, or after antiretroviral administration were matched by cell line and compared with tenofovir and emtricitabine only controls using the unpaired nonparametric $t$-test. The one-way analysis of variance test was used to assess interday variability. All statistical tests were two-sided, with significance set at 0.05. Data are reported as the mean \pm standard deviation and associated $P$ values. Statistical parameters were derived using GraphPad Prism version 5.04 (La Jolla, CA, USA).

\section{Results \\ Determination of uptake of tenofovir and emtricitabine}

Intracellular concentrations of tenofovir and emtricitabine in the five representative female genital tract cell lines are shown in Figure 1A and B. Differences in the uptake of tenofovir and emtricitabine across the cell lines versus controls were 
observed. Mean intracellular tenofovir concentrations were highest in HeLa cells $\left(13,022 \pm 4503 \mathrm{fmol} / 10^{6}\right.$ cells, $P=0.002)$ and Ect1/E6E7 cells $\left(10,778 \pm 3713 \mathrm{fmol} / 10^{6}\right.$ cells, $P=0.002$ ), representing a 32 -fold and 27 -fold increase, respectively, compared with tenofovir uptake by THP-1 macrophage control cells ( $403 \pm 183 \mathrm{fmol} / 10^{6}$ cells). Intracellular tenofovir concentrations were comparable between THP-1, BC-3, and TF-1 cells. Emtricitabine uptake was also highest in HeLa $\left(1152 \pm 587 \mathrm{fmol} / 10^{6}\right.$ cells, $P=0.002)$ and Ect1/E6E7 cells $\left(1143 \pm 582 \mathrm{fmol} / 10^{6}\right.$ cells, $P=0.002)$ compared with THP-1 (18.1 $\pm 12.0 \mathrm{fmol} / 10^{6}$ cells), BC-3 (19.5 $\pm 12.3 \mathrm{fmol} / 10^{6}$ cells $)$, and TF-1 cells (44.1 $\pm 36.7 \mathrm{fmol} / 10^{6}$ cells). Uptake in HeLa and Ect1/ E6E7 cells was 63-fold higher compared with THP-1 cells. The average percentage uptake of tenofovir for the five cell lines was higher than the uptake of emtricitabine $(4.85 \%$ of the tenofovir dose versus $0.07 \%$ of the emtricitabine dose). The median intracellular tenofovir and emtricitabine concentration across all five lines was 579 and $40 \mathrm{fmol} / 10^{6}$ cells, respectively.

\section{Determination of uptake of tenofovir and emtricitabine in the presence of ethinyl estradiol and/or etonogestrel}

Concentrations of intracellular tenofovir and emtricitabine when dosed prior to, simultaneously, and after ethinyl estradiol or etonogestrel exposure across the five female genital tract surrogate cell types are shown in Table 1.

\section{A}
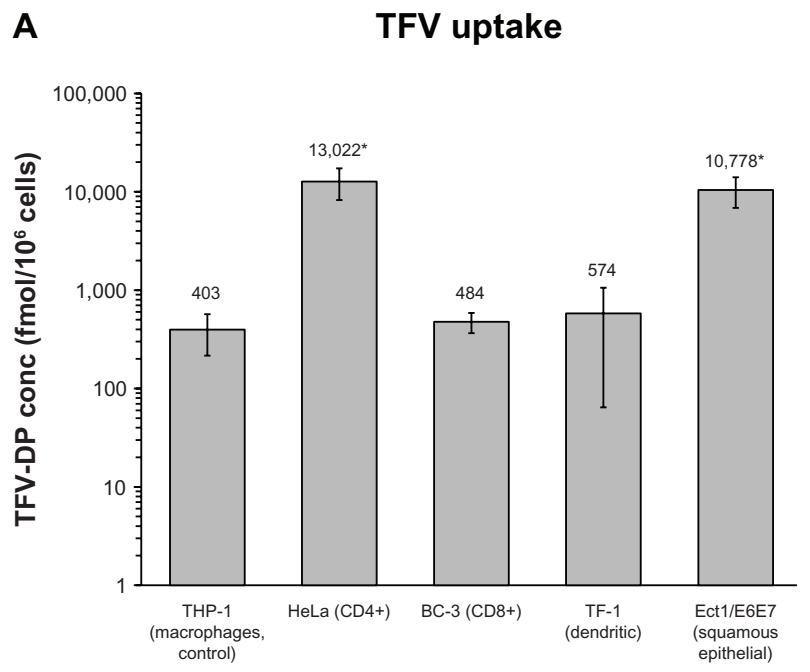

Uptake of tenofovir and emtricitabine prior to dosing with ethinyl estradiol or etonogestrel

BC-3 and TF-1 cells, when treated with tenofovir for one hour prior to adding ethinyl estradiol, demonstrated decreased intracellular tenofovir concentrations of $87 \%$ and $78 \%$, respectively, compared with tenofovir only controls. Similarly, when dosed with tenofovir prior to etonogestrel, intracellular tenofovir concentrations decreased by $87 \%$ in BC-3 cells and 79\% in TF-1 cells. No uptake effect was seen for THP-1, HeLa, or Ect1/E6E7 cells when dosed prior to ethinyl estradiol or etonogestrel. BC-3 and TF-1 cells also showed significantly decreased emtricitabine uptake when dosed prior to ethinyl estradiol (78\% and $85 \%$, respectively) and etonogestrel ( $69 \%$ and $96 \%$, respectively), with no differences in the remaining three cell lines.

\section{Uptake of tenofovir and emtricitabine}

with simultaneous dosing of ethinyl

estradiol or etonogestrel

Simultaneous dosing of tenofovir and exogenous hormones produced a mixture of decreased and increased tenofovir uptake. When ethinyl estradiol or etonogestrel was dosed concurrently with tenofovir in HeLa cells, a $57 \%$ and $65 \%$ decrease, respectively, in intracellular tenofovir concentrations was observed. TF-1 cellular uptake decreased by $35 \%$ and BC-3 cellular uptake decreased by $85 \%$ with simultaneous tenofovir/etonogestrel administration. For emtricitabine, the most pronounced effect was seen in BC-3 cells, where an 8.5-fold and 2.5-fold increase in intracellular emtricitabine

B FTC uptake

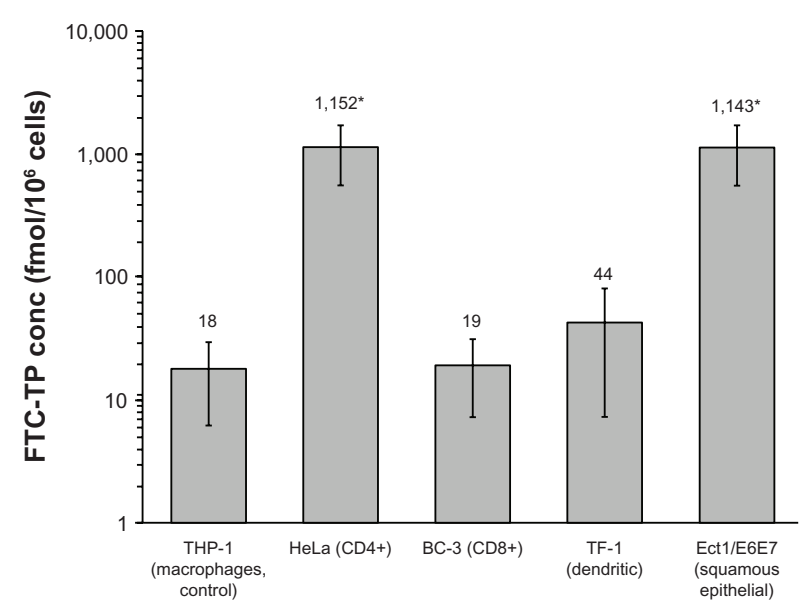

Figure I Uptake of TFV (A) and FTC (B) in five prominent cell types of the female genital tract. Concentrations of TFV-DP (A) and FTC-TP (B) in five cell lines representing the female genital tract. Cell lines areTHP-I (representing macrophages and control), HeLa (CD4+), BC-3 (CD8+), TF-I (dendritic), and Ect I/E6E7 (squamous epithelial cells) following a one hour incubation. Mean \pm standard deviation values are reported * indicates significantly different from control. Each cell line represents 6 replicates except the BC-3 and TF-I for TFV-DP which each had 5 replicates.

Abbreviations: TFV, tenofovir; TFV-DP, tenofovir diphosphate; FTC, emtricitabine; FTC-TP, emtricitabine triphosphate. 
Table I Mean \pm SD concentration of TFV and FTC in five cell types of the female genital tract when dosed prior to, simultaneously, and after ET or EE administration

\begin{tabular}{|c|c|c|c|c|c|c|c|}
\hline \multirow[t]{3}{*}{ Cell line } & \multicolumn{7}{|c|}{ Uptake of TFV (fmol/ $10^{6}$ cells) } \\
\hline & \multirow[t]{2}{*}{ TFV only } & \multicolumn{2}{|c|}{ TFV dosed I hr prior } & \multicolumn{2}{|c|}{ TFV dosed simultaneously } & \multicolumn{2}{|c|}{ TFV dosed I hr after } \\
\hline & & EE & ET & EE & ET & EE & ET \\
\hline THP-I & $393.6 \pm 19.9$ & $374.7 \pm 7.3$ & $377.1 \pm 8.9$ & $4 \mid 2.7 \pm 12.3$ & $358.9 \pm 18.3$ & $309.9 \pm 7.9 * *$ & $295.7 \pm 3.8^{* *}$ \\
\hline $\mathrm{BC}-3$ & $469.7 \pm 14.6$ & $61.9 \pm 1.5^{* *}$ & $63.2 \pm 1.7^{* *}$ & $489.7 \pm 18.5$ & $869.4 \pm 16.2^{* *}$ & $387.3 \pm 12.6^{* *}$ & $331.7 \pm 15.4^{* *}$ \\
\hline Ectl/E6E7 & $11,912 \pm 720.4$ & $|2,55| \pm 757.8$ & $14,945 \pm 3054$ & $11,539 \pm 283.6$ & $|I, 987 \pm| 79 \mid$ & $9457 \pm 687.2$ & $9076 \pm 1207$ \\
\hline HeLa & $18,456 \pm 2294$ & $7 \mathrm{I}, 680 \pm 4 \mathrm{I}, 798$ & $22,597 \pm 6524$ & $7999 \pm 2959 *$ & $6495 \pm 420.2^{* *}$ & $38,199 \pm 850 I^{*}$ & $82,369 \pm 36,250$ \\
\hline \multirow[t]{4}{*}{ TF-I } & $505.7 \pm 16.4$ & $1 \mathrm{II} .8 \pm 2.8^{* *}$ & $108.4 \pm 2.5^{* *}$ & $465.2 \pm 13.3$ & $330.6 \pm 10.0 * *$ & $315.6 \pm 10.7^{* *}$ & $266.3 \pm 11.0$ ** \\
\hline & \multicolumn{7}{|c|}{ Uptake of FTC (fmol/ $10^{6}$ cells) } \\
\hline & \multirow[t]{2}{*}{ FTC Only } & \multicolumn{2}{|c|}{ FTC dosed I hr prior } & \multicolumn{2}{|c|}{ FTC dosed simultaneously } & \multicolumn{2}{|c|}{ FTC dosed I hr after } \\
\hline & & EE & ET & EE & ET & EE & ET \\
\hline THP-I & $74.8 \pm 2.6$ & $78.8 \pm 3.0$ & $72.5 \pm 3.5$ & $64.1 \pm 1.6^{*}$ & $54.4 \pm 2.1 * *$ & $64.5 \pm 2.5^{*}$ & $77.4 \pm 3.0$ \\
\hline BC-3 & $35.7 \pm 2.5$ & $7.7 \pm 0.2^{* *}$ & $I I . I \pm 0.6 * *$ & $304.9 \pm 14.7^{* *}$ & $88.7 \pm 5.0 * *$ & $69.1 \pm 3.9 * *$ & $88.5 \pm 4.7 * *$ \\
\hline Ectl/E6E7 & $1357 \pm 211.9$ & $1228 \pm 54.6$ & $1110 \pm 38.1$ & $834.2 \pm 153.8^{* *}$ & $837.7 \pm 150.8^{*}$ & $1279 \pm 189.1$ & $1186 \pm 43.6$ \\
\hline HeLa & $200 \mid \pm 123.1$ & $2087 \pm 73.7$ & $1877 \pm 61.4$ & $2078.3 \pm 87.8$ & $186 \mid .1 \pm 116.2$ & $2011 \pm 33.4$ & $1870 \pm 84.9$ \\
\hline TF-I & $75.1 \pm 4.5$ & $1 \mathrm{I} . \mathrm{I} \pm 0.6 * *$ & $3.2 \pm 0.3^{* *}$ & $74.2 \pm 4.5$ & $33.7 \pm 2.4^{* *}$ & $62.0 \pm 4.2$ & $28.0 \pm 2.3 * *$ \\
\hline
\end{tabular}

Notes: THP-I, macrophages; BC-3, CD8+; EctI/E6E7, squamous epithelial; HeLa, CD4+; TF-I, dendritic cells; $*$ indicates $P<0.05 ; * *$ indicates $P<0.0$ I compared to TFV or FTC only.

Abbreviations: SD, standard deviation; EE, ethinyl estradiol; ET, etonogestrel; TFV, tenofovir; FTC, emtricitabine.

was observed following simultaneous dosing with ethinyl estradiol or etonogestrel, respectively. Uptake of emtricitabine by Ect1/E6E7 cells decreased by 39\% with ethinyl estradiol, while the uptake of emtricitabine by THP-1, Ect1/E6E7, and TF-1 cells decreased by $27 \%$ to $55 \%$ with etonogestrel.

Uptake of tenofovir and emtricitabine after dosing with ethinyl estradiol or etonogestrel

Significant alterations in intracellular tenofovir concentrations were observed when tenofovir was dosed one hour after incubation with ethinyl estradiol or etonogestrel. THP-1, BC-3, and TF-1 cells showed reductions in uptake ranging from $18 \%$ to $47 \%$ compared with tenofovir only controls. HeLa cells showed increased intracellular tenofovir concentrations of 2.1-fold and 4.5-fold with ethinyl estradiol or etonogestrel, respectively. Intracellular emtricitabine concentrations decreased $14 \%$ in THP- 1 cells and increased 1.9-fold in BC-3 cells when added after ethinyl estradiol. Emtricitabine also increased in BC-3 cells when added after etonogestrel, but decreased $63 \%$ in TF-1 cells.

\section{Uptake of tenofovir and emtricitabine when dosed prior, simultaneously, and after ethinyl estradiol and etonogestrel}

Intracellular tenofovir and emtricitabine concentrations by cell line when dosed prior to, simultaneously, and after ethinyl estradiol and etonogestrel together are shown in Figure 2. No differences in cellular uptake were noted for tenofovir in the THP-1, HeLa, or Ect1/E6E7 cell lines. For emtricitabine, there were also no differences in the HeLa and Ect1/E6E7 cell lines, but when dosed simultaneously in THP-1 cells, intracellular emtricitabine concentrations were $16 \%$ lower $(P<0.05)$ relative to the control group. The largest differences were seen in the BC-3 and TF- 1 cell lines. Results from these two cell lines for tenofovir and emtricitabine are shown in Figure 3. For both drugs, the most significant and consistent decreases were observed when dosed one hour prior to administration of EE/ET. The fold decreases in BC- 3 and TF-1 cells ranged from 5.9 to 6.6 for tenofovir and from 3 to 15 for emtricitabine, respectively.

\section{Influence of EE/ET on tenofovir and emtricitabine uptake over a 25 -fold range of antiretroviral doses} The significant difference (seen in Figure 2) in cellular uptake of tenofovir and emtricitabine, specifically for both BC-3 and TF-1 cells, lead to their evaluation of cellular uptake with additional antiretroviral doses in conjunction with hormonal contraceptives. In BC-3 cells, mean intracellular tenofovir concentrations increased approximately $275 \%$ when the dose was increased from $0.06 \mu \mathrm{g} / \mathrm{mL}$ to $0.3 \mu \mathrm{g} / \mathrm{mL}$ and increased approximately $372 \%$ when the dose increased from $0.3 \mu \mathrm{g} / \mathrm{mL}$ to $1.5 \mu \mathrm{g} / \mathrm{mL}$. Mean intracellular emtricitabine concentrations in BC-3 cells increased approximately $200 \%$ when the dosed increased from $0.09 \mu \mathrm{g} / \mathrm{mL}$ to 1.8 and increased approximately $35 \%$ when the dose increased from $1.8 \mu \mathrm{g} / \mathrm{mL}$ to $2.25 \mu \mathrm{g} / \mathrm{mL}$. Similar to BC-3 cells, tenofovir uptake by TF-1 cells increased approximately $330 \%$ and $324 \%$ when 

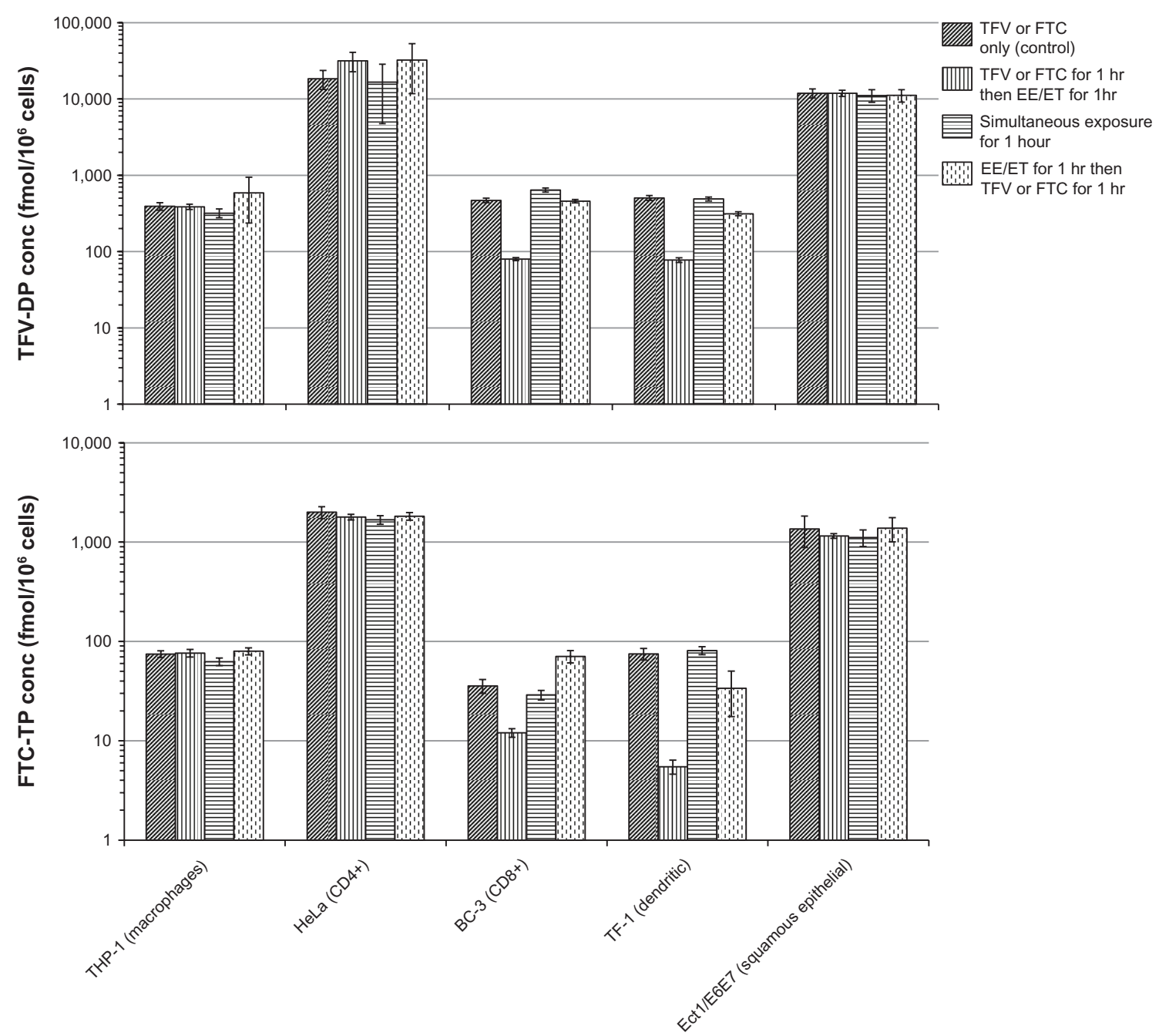

Figure 2 Intracellular TFV and FTC concentrations by cell line when dosed prior, simultaneously, and after EE and ET. TFV-DP (top panel) and FTC-TP (bottom panel) concentrations by cell type and dosing relative to EE and ET administration. Diagonal lines represent controls (TFV or FTC only incubated for I hour). Vertical lines represent TFV or FTC incubated for I hour, then EE and ET added for I hour. Horizontal lines represent TFV or FTC incubated simultaneously with EE and ET for I hour. Dashed vertical lines represent the cells incubated with EE and ET for I hour, then TFV or FTC were added for I hour.

Abbreviations: TFV, tenofovir; TFV-DP, tenofovir diphosphate; FTC, emtricitabine; FTC-TP, emtricitabine triphosphate; EE, ethinyl estradiol; ET, etonogestrel.

the dose increased to $0.3 \mu \mathrm{g} / \mathrm{mL}$ and $1.5 \mu \mathrm{g} / \mathrm{mL}$, respectively. In contrast with BC-3 cells, mean intracellular emtricitabine concentrations in TF- 1 cells increased only $24 \%$ when the dose was increased to $1.8 \mu \mathrm{g} / \mathrm{mL}$ and $228 \%$ when increased to $2.25 \mu \mathrm{g} / \mathrm{mL}$. Intraday and interday variability (coefficients of variation) of the uptake of tenofovir and emtricitabine by the cell lines were $2.5 \%$ to $69 \%$ and $6.9 \%$ to $62 \%$, respectively.

\section{Discussion}

This study evaluated the uptake of tenofovir and emtricitabine in vitro in representative cell types found in the female genital tract, and examined their uptake in the presence of hormonal contraceptives. Compared with the THP-1 control cell line, which represents macrophages, intracellular tenofovir and emtricitabine concentrations were increased in HeLa cells, which represent CD4+ T cells, and Ect1/E6E7 cells, a surrogate for squamous epithelial cells. Previous work has shown that these two cell lines are more rapidly invaded by HIV-1. ${ }^{30,31}$ In the presence of exogenous sex hormones, the most significant and consistent effect was observed for both tenofovir and emtricitabine when dosed one hour prior to EE/ET in BC-3 and TF-1 cells. These results indicate that differential uptake and phosphorylation of tenofovir and emtricitabine exists across major cellular subtypes in the female genital tract, and that sex hormones can influence cellular uptake and/or phosphorylation rates of these nucleoside analogs.

The five distinct surrogate female genital tract cells lines we evaluated in the absence of exogenous hormones achieved median intracellular tenofovir and emtricitabine concentrations of 579 and $40 \mathrm{fmol} / 10^{6}$ cells, respectively, 

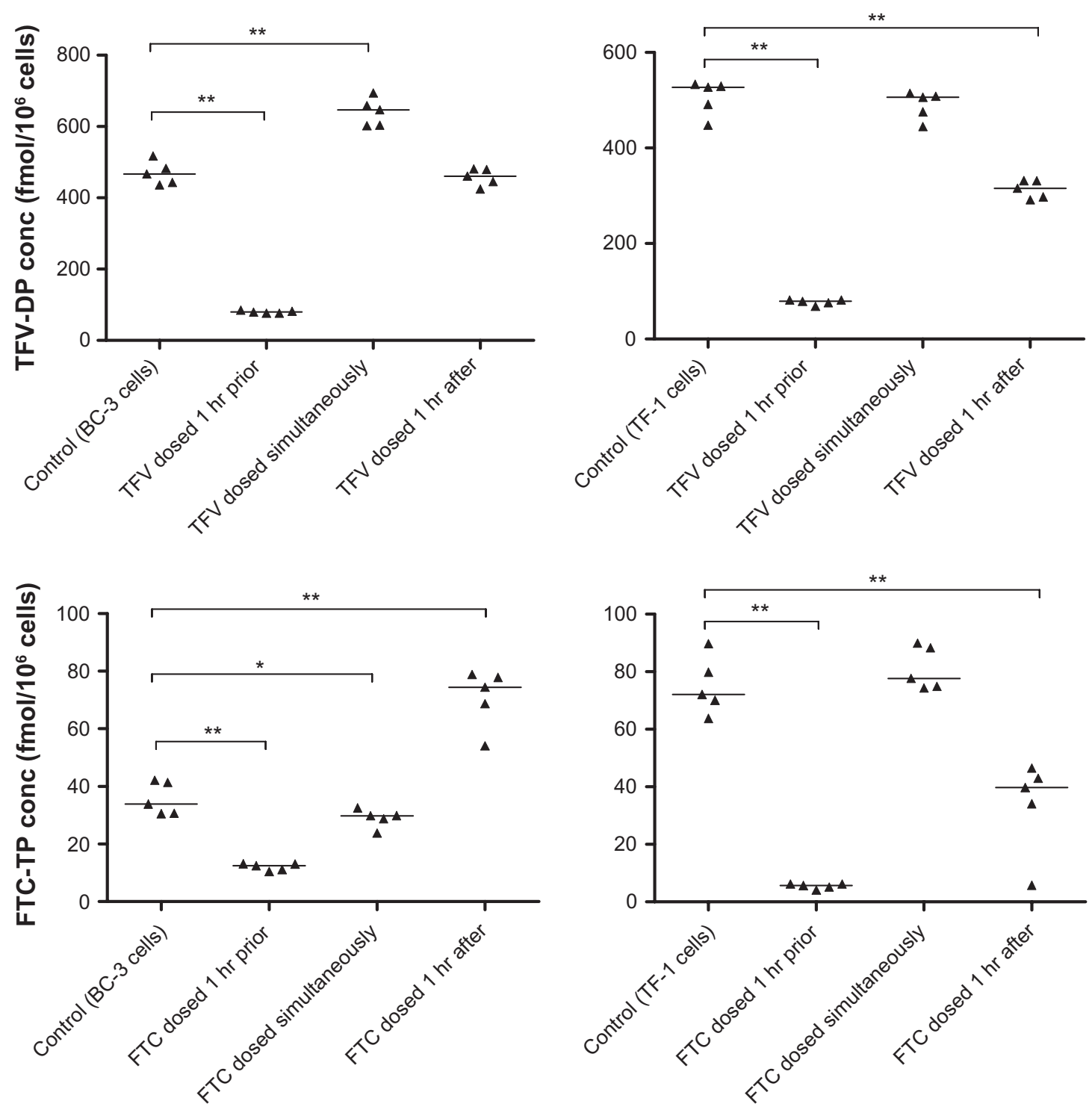

Figure 3 Intracellular TFV and FTC concentrations when dosed prior, simultaneously, and after EE and ET in BC-3 and TF-I cell lines. Top panels represent TFV-DP concentrations in BC-3 (left) and TF-I (right) cell lines. Bottom panels depict FTC-TP concentrations in BC-3 (left) and TF-I (right) cell lines. Double (**) and single (*) asterisks indicate $P<0.0 \mathrm{I}$ and 0.05 , respectively, vs control.

Abbreviations: TFV, tenofovir; TFV-DP, tenofovir diphosphate; FTC, emtricitabine; FTC-TP, emtricitabine triphosphate; EE, ethinyl estradiol; ET, etonogestrel.

which for tenofovir is considerably higher than what has been reported to reduce viral transmission from male patients by $90 \%$ in plasma peripheral blood mononuclear cells $\left(15.6 \mathrm{fmol} / 10^{6}\right.$ cells). ${ }^{32}$ However, our data, were derived from in vitro cellular uptake studies using surrogate female genital tract cell lines and not plasma peripheral blood mononuclear cells. An in vivo study that collected steady-state HIV-1 negative cervical cell samples reported a tenofovir diphosphate concentration of $337 \mathrm{fmol} / 10^{6}$ cells and an emtricitabine triphosphate concentration of $4300 \mathrm{fmol} / 10^{6}$ cells. ${ }^{33}$ Our median emtricitabine triphosphate concentration across the two cell lines with the highest uptake (HeLa and Ect1/E6E7) was 934 fmol/10 cells. Importantly, our goal was to examine uptake across these cell lines and assess the impact that exogenous hormones may have on uptake, so our in vitro results are not necessarily comparable with clinical data with regard to exposures required for prevention of transmission.

Penetration of nucleoside reverse transcriptase inhibitors into the female genital tract is dictated by facilitated diffusion and active transport. ${ }^{34}$ One potential explanation for differences in observed antiretroviral uptake is that various cell types in the female genital tract may express transporters differently and these transporters likely have varying degrees of functional activity. The data suggest that uptake of tenofovir occurs through organic anion 
transporter (OAT) 1 and 3 while uptake of emtricitabine occurs through organic cation transporter (OCT) 1. ${ }^{35,36}$ Expression and/or activity of these transporters relative to cells vulnerable to HIV-1 transmission in the female genital tract have not been extensively evaluated. However, these transporters can be manipulated by cyclic nucleotides and steroids. ${ }^{37-39}$ Progesterone upregulates OAT 1, which is used by tenofovir for entry into the cell, and downregulates OCT 1 and multidrug resistance protein (MRP) 1, 4, and 5, which is used by emtricitabine for entry into the cell. Through downregulation of MRP, the cellular efflux of tenofovir diphosphate and emtricitabine triphosphate is inhibited, thus potentially increasing the intracellular anabolites. Estrogen downregulates OAT 1 and 3 and OCT 1, which are both required by tenofovir and emtricitabine. Coadministration of ethinyl estradiol, etonogestrel, tenofovir, and emtricitabine may alter uptake of tenofovir and emtricitabine and potentially affect clinical outcomes. Our results suggest that intracellular tenofovir and emtricitabine concentrations, particularly in $\mathrm{BC}-3$ and $\mathrm{TF}-1$ cells, were altered in the presence of exogenous sex hormones. The percent uptake of tenofovir in the presence of EE/ET was similar as tenofovir doses increased. However, percent uptake of emtricitabine in the presence of EE/ET was variable as emtricitabine doses increased. Taken together, our data support the need for future in vivo studies designed to assess whether these effects occur in patients, and if so, their clinical significance.

The effect of synthetic sex hormones on the intracellular phosphorylation of nucleosides has not been directly studied. However, studies have been conducted with some of the intracellular enzymes responsible for phosphorylation and dephosphorylation of both tenofovir and emtricitabine and other nucleoside reverse transcriptase inhibitors in the presence of sex hormones. Human adenylate kinase, which phosphorylates tenofovir to its monophosphate anabolite, is not affected by estrogen, ${ }^{40}$ indicating that the difference in uptake and phosphorylation of tenofovir is more closely regulated by transporters for cell entry or the enzyme responsible for the second stage of phosphorylation, ie, nucleotide diphosphate kinase. The effect of the three enzymes responsible for the phosphorylation of emtricitabine has not been evaluated with ethinyl estradiol and etonogestrel in vitro. However, the enzyme (5'-nucleosidase) responsible for the dephosphorylation of emtricitabine monophosphate to emtricitabine shows altered enzymatic activity when dosed with estradiol and progesterone in homogenated rat brain and liver. ${ }^{41}$ The liver showed decreased enzymatic activity in the presence of both synthetic hormones, while estradiol in the brain increased the activity of the enzyme. A decrease in the efflux of emtricitabine would theoretically lead to an increase in the intracellular phosphorylated moiety. Another study has shown that high physiologically relevant concentrations of progesterone decrease $(25 \%-$ $68 \%$ ) the phosphorylated forms of nucleoside reverse transcriptase inhibitors, while estrogen shows no significant change. ${ }^{42}$ These data suggest that intracellular tenofovir and emtricitabine may be altered by exogenous progesterone, which is consistent with our results, but the direction and magnitude of the change is variable.

There are some limitations to the studies we have conducted. Antiretroviral uptake was determined in only one female genital tract cell line for each cell type, and immortal cells were used. Our findings should be validated against additional cell lines representing these and other cell subtypes from the female genital tract and by primary female genital tract cells obtained in a clinical setting. Of note, our experiments were conducted using a one-hour incubation time. Earlier studies evaluating the mechanistic and kinetic activity of antiretrovirals or hormones suggest that one-hour incubations are sufficient for uptake of antiretrovirals and synthetic hormones. ${ }^{43-45}$ However, varying incubation times may be needed to account for any changes in cellular antigen, receptor, and/or transporter activity potentially caused by the addition of tenofovir, emtricitabine, ethinyl estradiol, or etonogestrel. Only one concentration of ethinyl estradiol and etonogestrel was included in the cell medium. Evaluating various doses of ethinyl estradiol and etonogestrel would determine if uptake of tenofovir and emtricitabine into the surrogate female genital tract cells is dependent on the functional activity of transporters induced and inhibited by ethinyl estradiol and etonogestrel. Importantly, the concentrations of ethinyl estradiol and etonogestrel used represent the plasma $\mathrm{C}_{\max }$ in humans. Intravaginal concentrations could be considerably different and vary from woman to woman, so differences in the magnitude of effect at the local site may still exist. Concentrations of ethinyl estradiol and etonogestrel have been quantified in the female genital tract following use of the NuvaRing contraceptive. ${ }^{46}$ The mean female genital tract concentrations (from the cervical region) of ethinyl estradiol after one cycle (17-21 days) was $68 \mathrm{pg} / 0.5 \mathrm{~g}$ tissue, which assuming $1 \mathrm{~g}$ is equivalent to $1 \mathrm{~mL}$, is approximately $75 \%$ higher than the concentration used in this study $(34.7 \mathrm{pg} / \mathrm{mL})$. The mean etonogestrel concentration was $327 \mathrm{pg} / 0.5 \mathrm{~g}$ tissue, which is approximately $62 \%$ lower compared with our study $(1716 \mathrm{pg} / \mathrm{mL})$. Etonogestrel, which is uniquely found in the 
hormonal contraceptive NuvaRing, was the only progesterone studied. Most hormonal contraceptives use a combination of ethinyl estradiol and different synthetic progesterones, such as norethindrone, norgestimate, or levonorgestrel. Given that the mechanism of action of etonogestrel is similar to that of other synthetic progesterones, a similar effect on cellular uptake of tenofovir and emtricitabine would be expected.

In conclusion, our data suggest that intracellular concentrations of tenofovir and emtricitabine differ across surrogate cell types of the female genital tract and these concentrations may be altered in the presence of exogenous sex hormones. Furthermore, in vitro studies using THP-1 cells only to make in vivo correlations between antiretroviral dosing and intracellular concentrations in the female genital tract may not be sufficient. The design of future PrEP studies should factor in patient use of hormonal contraceptives, because exogenous hormones can change the cellular makeup of the cervical mucus and endometrium. These changes shift the prevalence of certain cell types in the female genital tract and may alter the total amount of intracellular drug present. ${ }^{47}$ As tenofovir, emtricitabine, and additional PrEP agents are clinically evaluated in women, the pharmacologic consequences of exogenous hormone therapy should be considered.

\section{Acknowledgments}

This work was conducted with the support of grant OPP1002972 from the Bill and Melinda Gates Foundation, grant P30AI050409 by the Emory Center for AIDS Research funded through the National Institute of Allergy and Infectious Diseases, grant UL1RR025008 from the Atlanta Clinical and Translational Science Institute funded through the National Center for Research Resources, and grant U01AI103408-01 from the Women Interagency HIV Cohort Study. AMJ received funding from the University of Alabama at Birmingham Office of Diversity and Equity by the Comprehensive Minority Student Development Program at University of Alabama at Birmingham.

\section{Disclosure}

The authors report no conflicts of interest in this work.

\section{References}

1. Al-Jabri AA, Alenzi FQ. Vaccines, virucides and drugs against HIV/ AIDS: hopes and optimisms for the future. Open AIDS J. 2009;3:1-3.

2. Buchbinder $\mathrm{S}$. The epidemiology of new HIV infections and interventions to limit HIV transmission. Top HIV Med. 2009;17:37-43.

3. Abdool-Karim Q, Abdool-Karim SS, Frohlich JA, et al. Effectiveness and safety of tenofovir gel, an antiretroviral microbicide, for the prevention of HIV infection in women. Science. 2010;329:1168-1174.
4. Anderson P, Liu A, Grant R, et al. Intracellular tenofovir-DP concentrations associated with PrEP efficacy in MSM from iPrEx. Abstract 31B presented at the 19th Conference on Retroviruses and Opportunistic Infections, Seattle, WA, March 3-6, 2012.

5. Curran K, Baeten JM, Coates TJ, et al. HIV-1 Prevention for HIV-1 serodiscordant couples. Curr HIV/AIDS Rep. 2012;9:160-170.

6. Celum C, Baeten JM. Tenofovir-based pre-exposure prophylaxis for HIV prevention: evolving evidence. Curr Opin Infect Dis. 2012;25: 51-57.

7. McEnery R. Oral tenofovir arm of VOICE trial discontinued early. IAVI Rep. 2011;15:21.

8. [No authors listed]. Early end for FEM-PrEP HIV prevention trial. AIDS Patient Care STDs. 2011;25:383.

9. Hendrix C, Minnis A, Guddera V, et al. MTN-001: a phase 2 crossover study of daily oral and vaginal TFV in healthy, sexually active women results in significantly different product acceptability and vaginal tissue drug concentrations. Abstract $35 \mathrm{LB}$ presented at the $18 \mathrm{th}$ Conference on Retroviruses and Opportunistic Infections, Boston, MA, February 27-March 5, 2011.

10. Donnell D, Baeten J, Hendrix C, et al. Tenofovir disoproxil fumarate drug levels indicate PrEP use is strongly correlated with HIV-1 protective effects: Kenya and Uganda. Abstract 30 presented at the 19th Conference on Retroviruses and Opportunistic Infections, Seattle, WA, March 3-6, 2012.

11. Kearney BP, Mathias A. Lack of effect of tenofovir disoproxil fumarate on pharmacokinetics of hormonal contraceptives. Pharmacotherapy. 2009;29:924-929.

12. Cihlar T, Ray AS. Nucleoside and nucleotide HIV reverse transcriptase inhibitors: 25 years after zidovudine. Antiviral Res. 2010;85: 39-58.

13. Robbins BL, Srinivas RV, Kim C, et al. Anti-human immunodeficiency virus activity and cellular metabolism of a potential prodrug of the acyclic nucleoside phosphonate 9-R-(2-phosphonomethoxypropyl) adenine (PMPA), Bis(isopropyloxymethylcarbonyl)PMPA. Antimicrob Agents Chemother. 1998;42:612-617.

14. Anderson PL, Kiser JJ, Gardner EM, et al. Pharmacological considerations for tenofovir and emtricitabine to prevent HIV infection. J Antimicrob Chemother. 2011;66:240-250.

15. Pudney J, Quayle AJ, Anderson DJ. Immunological microenvironments in the human vagina and cervix: mediators of cellular immunity are concentrated in the cervical transformation zone. Biol Reprod. 2005;73:1253-1263.

16. Hajjar AM, Lewis PF, Endeshaw Y, et al. Efficient isolation of human immunodeficiency virus type 1 RNA from cervical swabs. J Clin Microbiol. 1998;36:2349-2352.

17. Cu-Uvin S, Caliendo AM, Reinert S, et al. Effect of highly active antiretroviral therapy on cervicovaginal HIV-1 RNA. AIDS. 2000;14: 415-421.

18. Reddy YS, Kashuba A, Gerber J, et al. Roundtable report: importance of antiretroviral drug concentrations in sanctuary sites and viral reservoirs. AIDS Res Hum Retroviruses. 2003;19:167-176.

19. Brenchley JM, Douek DC. The mucosal barrier and immune activation in HIV pathogenesis. Curr Opin HIV AIDS. 2008;3:356-361.

20. Haase AT. The pathogenesis of sexual mucosal transmission and early stages of infection: obstacles and a narrow window of opportunity for prevention. AIDS. 2001;15:S10-S11.

21. Blankson JN, Persaud D, Siliciano RF. The challenge of viral reservoirs in HIV-1 infection. Annu Rev Med. 2002;53:557-593.

22. Mikovits JA, Raziuddin, Gonda M, et al. Negative regulation of human immune deficiency virus replication in monocytes. Distinctions between restricted and latent expression in THP-1 cells. J Exp Med. 1990;171: 1705-1720.

23. Hladik F, Sakchalathorn P, Ballweber L, et al. Initial events in establishing vaginal entry and infection by human immunodeficiency virus type-1. Immunity. 2007;26:257-270.

24. Chang F, Steelman LS, McCubrey JA. Raf-induced cell cycle progression in human TF-1 hematopoietic cells. Cell Cycle. 2002;1:220-226. 
25. Iversen AK, Larsen AR, Jensen T, et al. Distinct determinants of human immunodeficiency virus type 1 RNA and DNA loads in vaginal and cervical secretions. J Infect Dis. 1998;177:1214-1220.

26. Africander D, Louw R, Verhoog N, et al. Differential regulation of endogenous pro-inflammatory cytokine genes by medroxyprogesterone acetate and norethisterone acetate in cell lines of the female genital tract. Contraception. 2011;84:423-435.

27. Viread [package insert]. Foster City, CA: Gilead Sciences Inc; 2011.

28. Emtriva [package insert]. Foster City, CA: Gilead Sciences Inc; 2011.

29. Timmer CJ, Mulders TM. Pharmacokinetics of etonogestrel and ethinylestradiol released from a combined contraceptive vaginal ring. Clin Pharmacokinet. 2000;39:233-242.

30. Coombs RW, Wright DJ, Reichelderfer PS, et al. Variation of human immunodeficiency virus type 1 viral RNA levels in the female genital tract: Implications for applying measurements to individual women. J Infect Dis. 2001;184:1187-1191.

31. Hladik F, McElrath MJ. Setting the stage: host invasion by HIV. Nat Rev Immunol. 2008;8:447-457.

32. Anderson P, Liu A, Buchbinder S, et al. Intracellular tenofovir-DP concentrations associated with PrEP efficacy in MSM from iPrEx. Abstract 31LB presented at the 19th Conference on Retroviruses and Opportunistic Infections. Seattle, WA, March 3-6, 2012.

33. Anderson P, Meditz A, Zheng JH, et al. Cellular pharmacology of tenofovir and emtricitabine in blood, rectal, and cervical cells from HIV- volunteers. Abstract 587 presented at the 19th Conference on Retroviruses and Opportunistic Infections. Seattle, WA, March 3-6, 2012.

34. Taylor S, Pereira AS. Antiretroviral drug concentrations in the semen of HIV-1 infected men. Sex Transm Inf. 2001;77:4-11.

35. Cihlar T, Ray AS, Laflamme G, et al. Molecular assessment of the potential for renal drug interactions between tenofovir and HIV protease inhibitors. Antivir Ther. 2007;12:267-272.

36. Nakatani-FreshwaterT, Taft DR. Renal excretion of emtricitabine I: effects of organic anion, organic cation, and nucleoside transport inhibitors on emtricitabine excretion. J Pharm Sci. 2008;97:5401-5410.

37. Wielinga PR, van der Heijden I, Reid G, et al. Characterization of the MRP4- and MRP5-mediated transport of cyclic nucleotides from intact cells. J Biol Chem. 2003;278:17664-17671.
38. Hayer-Zillgen M, Brüss M, Bönisch H. Expression and pharmacological profile of the human organic cation transporters hOCT1, hOCT2 and hOCT3. Br J Pharmacol. 2002;136:829-836.

39. Ljubojevic M, Herak-Kramberger CM, Hagos Y, et al. Rat renal cortical OAT1 and OAT3 exhibit gender differences determined by both androgen stimulation and estrogen inhibition. Am J Physiol Renal Physiol. 2004;287:F124-F138.

40. Roghmann M-C, Skinner KA, Hilf R. Effects of estradiol and tamoxifen on creatine kinase in rodent mammary carcinomas. Cancer Res. 1987;47:1348-1353.

41. Garcia MV, Cabezas JA, Perez-Gonzalez MN. Effects of oestradiol, testosterone and medroxyprogesterone on subcellular fraction marker enzyme activities from rat liver and brain. Comp Biochem Physiol B. $1985 ; 80: 347-354$

42. Anderson PL, King T, Zheng JH, et al. Cytokine and sex hormone effects on zidovudine- and lamivudine-triphosphate concentrations in vitro. J Antimicrob Chemother. 2008;62:738-745.

43. Si-Mohamed A, Kazatchkine MD, Heard I, et al. Selection of drugresistant variants in the female genital tract of human immunodeficiency virus type 1-infected women receiving antiretroviral therapy. J Infect Dis. 2000;182:112-122.

44. Okada M, Suzuki A, Mizuno K, et al. Effects of 17 beta-estradiol and progesterone on migration of human monocytic THP-1 cells stimulated by minimally oxidized low-density lipoprotein in vitro. Cardiovasc Res. 1997;34:529-535.

45. Harms C, Lautenschlager M, Bergk A, et al. Differential mechanisms of neuroprotection by 17 beta-estradiol in apoptotic versus necrotic neurodegeneration. J Neurosci. 2001;21:2600-2609.

46. Roumen FJ, Dieben TO. Comparison of uterine concentrations of ethinyl estradiol and etonogestrel after use of a contraceptive vaginal ring and an oral contraceptive. Fertil Steril. 2006;85:57-62.

47. Rivera R, Yacobson I, Grimes D. The mechanism of action of hormonal contraceptives and intrauterine contraceptive devices. Am J Obstet Gynecol. 1999;181:1263-1269.
Journal of Experimental Pharmacology

\section{Publish your work in this journal}

The Journal of Experimental Pharmacology is an international, peerreviewed, open access journal publishing original research, reports, reviews and commentaries on all areas of laboratory and experimental pharmacology. The manuscript management system is completely online and includes a very quick and fair peer-review system.

\section{Dovepress}

Visit http://www.dovepress.com/testimonials.php to read real quotes from published authors. 